\title{
A new treatment of radiation behaviour beyond one-body observables
}

\author{
Koji Niita ${ }^{1, a}$, Yosuke Iwamoto ${ }^{2}$, Tatsuhiko Sato $^{2}$, Hiroshi Iwase $^{3}$, Norihiro Matsuda ${ }^{2}$, \\ Yukio Sakamoto ${ }^{2}$, and Hiroshi Nakashima ${ }^{2}$ \\ Reasearch Organization for Information Science \& Technology (RIST), Tokai, Naka, Ibaraki 319-1106, Japan \\ 2 Japan Atomic Energy Agency (JAEA), Tokai, Naka, Ibaraki 319-1195, Japan \\ 3 High Energy Accelerator Research Organization (KEK), Oho, Tsukuba, Ibaraki 305-0801, Japan
}

\begin{abstract}
We propose a new treatment of radiation behaviour in transport calculations by introducing an event generator model in which we combine the nuclear data and the reaction models so as to trace all correlations of ejectiles keeping the energy and momentum conservation in a collision. By this new model, we can estimate the fluctuations around the mean values of one-body observables, for example, the deposit energy distribution in a cell, which cannot be obtained by the transport calculations based on the Boltzmann equation with the nuclear data.
\end{abstract}

\section{Introduction}

Nuclear data are extensively used in Monte Carlo transport calculations to analyze the radiation behaviour in various fields such as accelerator facilities, space radiation, and radiotherapy. Most of the Monte Carlo transport calculations are based on Boltzmann equation for one-body phase space distribution of the transport particles. In such transport calculations, one can obtain only the mean value of the onebody observables in the phase space, e.g., heat, flux, and dose, but not the fluctuations around the mean value, since the Boltzmann equation, nor the nuclear data has information on the two-body and higher order correlations which determine the fluctuation around the mean value.

Recently, however, the higher order quantities, i.e., the fluctuations around the mean values of the one-body observables are often required. A typical example for such a correlated quantity is the deposit energy distribution in a cell, which is necessary to estimate the response function of detectors, the single event upset probability of semiconductor memory cells and the radiation effects in a micro-dosimetric treatment. The solution of the Boltzmann equation cannot describe the distribution but only the mean value. Furthermore, Monte Carlo calculations using the nuclear data cannot deal with these quantities, since the nuclear data includes only the inclusive one-body cross sections but no information of the correlations.

We have therefore developed a new treatment of radiation behaviour in the transport calculations by introducing an event generator model, in which we have combined the nuclear data and the reaction models so as to trace all higher correlations of ejectiles keeping the energy and momentum conservation in a collision.

\section{Boltzmann equation vs. event generator}

For high energy transport calculations, the nuclear reaction models are commonly used instead of the evaluated nuclear data to describe the ejectiles from each nuclear reaction, since there is not enough evaluated nuclear data for the high energy reactions. The reaction models usually generate each event keeping the energy and momentum by the Monte Carlo method. In this sense, the reaction model is called as "Event Generator". Therefore we can extract any information from the transport calculations with such reaction models. On the other hand, the transport calculations based on the Boltzmann equation with the nuclear data, e.g., MCNP-type calculations, have no such concept of "Event". For the actual numerical solution of the Boltzmann equation, one usually employs the test particle method where the one-body phase-space distribution function is evaluated by integrating the test particle tracks in the phase-space. In this numerical calculation, things are going on in a very similar manner as in the high energy transport calculation with the event generator. However, there is no "Event" in this type of calculations. One history in this type of calculation has no physical meaning but gives us only a statistical weight. The observables are obtained after averaged out the statistical weight. There is no physical meaning in the distribution around the mean value but only the statistical variance. However, the distribution around the mean value calculated in the high energy transport code with the event generator mode is a real physical quantity, which is often required as mentioned above.

\section{Reaction models in PHITS}

We have developed a particle and heavy ion transport Monte Carlo code system, PHITS [1], for general purposes. PHITS has three important ingredients which enable us to simulate various nuclear reactions, hadron-nucleus reactions with energy up to $200 \mathrm{GeV}$, nucleus-nucleus collisions from $10 \mathrm{MeV} / \mathrm{u}$ up to $100 \mathrm{GeV} / \mathrm{u}$ and reactions of low energy neutron down to $10^{-5} \mathrm{eV}$. For this, PHITS employs three reaction models, a hadron cascade model JAM [2], a molecular dynamics model JQMD [3] for nucleus-nucleus collisions, and a reaction model based on the evaluated nuclear data for low energy neutrons and photons in the same manner as in MCNP4C [4]. JAM and JQMD are reaction models which satisfy the event generator mode but the MCNP part for low energy neutron is not an event generator mode. Thus two fields 
are mixed up in PHITS. In order to estimate the higher order quantities such as the deposit energy distribution for a whole energy range in PHITS, we have developed a new treatment of collisional processes for low energy neutrons.

By using the evaluated nuclear data, if it could include all cross sections of the ejectiles, we cannot construct an event by the Monte Carlo method except for the elastic channel, since the evaluated nuclear data has no information about correlation between ejectiles of a collision. Let us consider a typical example of this, i.e., neutron induced $(n, 2 n)$ reaction channel. In the MCNP-type calculation, the momentum of the first outgoing neutron is determined according to the nuclear data for the inclusive neutron spectra. The momentum of the second outgoing neutron is also determined in the same procedure independently of the first neutron. Thus, for example, the following situation can be happened; a neutron with the incident energy $19 \mathrm{MeV}$ hits a nucleus and produces the first neutron with energy of $12 \mathrm{MeV}$ and the second with $15 \mathrm{MeV}$. In this case, the total energy and momentum is violated in this event. This is due to a lack of information about the correlation in the evaluated nuclear data. In the MCNP-type calculation, however, the total energy and momentum is conserved in terms of the expectation values obtained by the integration with the one-body phase-space distributions, which is realized after averaging out the observables by the test particle tracks in the actual numerical simulation.

One possibility to construct an event for low energy neutron collision might be to make a pure theoretical model to describe the collision. However, it is almost hopeless since the cross section of neutron in the low energy below $20 \mathrm{MeV}$ has a lot of resonance structure and all of the resonances cannot be described purely by a theoretical model. Therefore, we have developed a new treatment of collisional processes for low energy neutrons by combining the evaluated nuclear data and a reaction model in the following way.

\section{Event generator mode in PHITS}

The evaluated nuclear data for the low energy neutron includes the information of the total cross section, the channel cross sections of capture, elastic, inelastic, and $\left(\mathrm{n}, \mathrm{Nn}^{\prime}\right)$ the inclusive double differential cross section of outgoing neutrons. From this information, the energy and momentum of all ejectiles including the residual nucleus are not determined uniquely, since in particular the information on the correlation is not available. Therefore, we have developed a model to determine the energy and momentum of all ejectiles, event by event, by using the information of the nuclear data and combining a special statistical decay model.

At first, we use the total cross section and the channel cross sections from the evaluated nuclear data to choose a channel of the collision. We categorize the channels by the number of outgoing neutrons. For the channel which does not produce neutrons, that we call "capture" channel, the excitation energy and momentum of the captured nucleus is determined uniquely from the incident energy of neutron and target nucleus. For the decay process of this excited nucleus, we apply a special statistical decay model, in which the decay width of neutron is assumed to be zero for the capture channel.
Considering the charged particle decay and photon decay in a statistical way, we can determine all energy and momentum of ejectiles and residual nucleus for the capture channel.

For the elastic channel, which is one of the channels categorized by one outgoing neutron, we choose the scattering angle of outgoing neutron by the Monte Carlo method according to the evaluated nuclear data. We can then determine the neutron energy and the momentum of the recoil nucleus uniquely by the kinematics of the elastic collision.

For the inelastic channel $\left(n, n^{\prime}\right)$, which is also a channel with one outgoing neutron, we first choose the momentum of the outgoing neutron according to the double differential cross sections of the evaluated nuclear data by Monte Carlo method. Using the kinematics of this emission, we can uniquely determine the excitation energy and the momentum of the residual nucleus. We then apply the same statistical decay procedure as in the capture channel without neutron decay width since there is no more neutron emission after the first neutron emission for this channel.

Finally for $\left(\mathrm{n}, \mathrm{Nn}^{\prime}\right)$ channel, which is a channel with $\mathrm{N}$ outgoing neutrons, we choose the momentum of the first outgoing neutron in the same way as in the $\left(n, n^{\prime}\right)$ case. After one nucleon emission, we apply only neutron decay procedure to the excited nucleus until $\mathrm{N}$ nucleons are emitted. Once $\mathrm{N}$ neutrons are emitted, the special decay procedure without neutron decay width is applied.

Using the above procedure, we can treat a low energy neutron collision as an "Event", keeping the energy and momentum conservation and preserving the channel cross sections and the inclusive neutron double differential cross sections, which are given by the evaluated nuclear data. The validation of the event generator mode in the PHITS code is presented in the other paper of these proceedings [5].

\section{Example of event generator mode}

Thanks to the event generator mode in PHITS for low energy neutron transport phenomena, we can calculate many new quantities which cannot be obtained by MCNP-type calculation, e.g., kinetic energy distribution of residual nuclei and charged particles, two-particle correlation, and so on. Here we show an example of the event generator mode in PHITS, i.e., deposit energy distribution in a cell, which is a typical quantity beyond one-body observable. This quantity is well known as a pulse height tally in MCNP. However, this is a conceptually wrong quantity to be treated in the MCNP-type calculation as discussed before. As mentioned in the MCNP manual, this tally is very restricted to use for neutron transport in MCNP. Namely, this tally does not work if a collision produces multiple neutrons.

Figure 1 shows the deposit energy distribution in a thin Si tip irradiated by $19 \mathrm{MeV}$ mono energetic neutrons. The thickness of the Si tip is $3 \mu \mathrm{m}$. If we calculate the deposit energy by using the Kerma factor in MCNP-type calculation, we get a delta-function-like single peak at $100 \mathrm{eV}$ with almost no distribution. However, figure 1 shows broad distribution obtained by the event generator mode in PHITS, where the deposit energy is estimated from the ionization losses of the charged particles and the recoil nuclei. This distribution is 


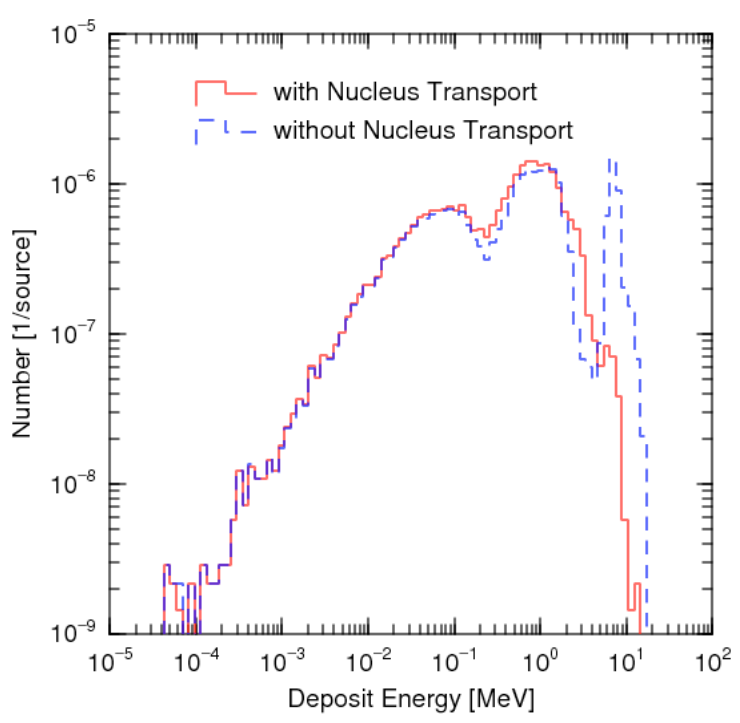

Fig. 1. Deposit energy distribution in a thin $(3 \mu \mathrm{m}) \mathrm{Si}$ tip irradiated by mono energetic neutron at $19 \mathrm{MeV}$. The solid (red) line is the result of the event generator mode of PHITS with nucleus transport, and the dashed (blue) line is the result of a local approximation, i.e., without nucleus transport.

not a statistical fluctuation in the Monte Carlo numerical calculation but a physical quantity to be considered. This distribution is also very important to estimate the single event upset probability of semiconductor memory cells, since only the events which induce the deposit energy greater than a certain threshold energy contribute to the error.

In figure 1, we have plotted two distributions, solid line and dashed line. The dashed line was obtained by the local approximation (Kerma approximation), in which all charged particles and recoil nuclei produced in neutron induced reactions are assumed to be stopped and deposit their kinetic energy at the collision point. This approximation is valid under the condition that the range of charged particles is much smaller than the size of the system. For this example, both are comparable since the range of charged particles is of the order of $\mu \mathrm{m}$, while the thickness of $\mathrm{Si}$ is $3 \mu \mathrm{m}$. Therefore we transported the charged particles and the recoil nuclei in the PHITS calculation without the local approximation. The result is shown by the solid line in figure 1. This shows that the highest peak of the dashed line has disappeared. This means that some part of the charged particles or the recoil nuclei (mainly alpha particles for this case) can escape from Si tip to the outside.

This example indicates two important points. The first is that the deposit energy distribution in a cell is well described by the event generator mode in PHITS even for low energy neutron transport below $20 \mathrm{MeV}$. The second is that the local approximation is not valid for the system whose size is comparable to the range of the transport charged particles and recoil nuclei.

\section{Conclusion}

We have proposed a new treatment of radiation behaviour in the particle and heavy ion transport code system PHITS by introducing an event generator model. In the event generator mode of PHITS, we combine the evaluated nuclear data and the reaction models to describe all the ejectiles of a collision keeping the energy and momentum conservation. Using this new model, we can estimate new quantities which are related to the higher order correlations beyond one-body observable. We have shown an example of such observable, the deposit energy distribution in a cell, which cannot be obtained by the MCNP-type transport calculation based on the Boltzmann equation with the evaluated nuclear data.

The existing nuclear data bases include many cross sections such as total cross section, channel cross section, double differential cross section of ejectile, and so on. These are all one-body inclusive data, while there is almost no data for the correlations between ejectiles. One reason of this is that huge storage is necessary to compile the correlation data particularly for higher energy, since the combination of the ejectiles is drastically growing up as energy is increasing. Another is that there is no need for the correlation data as far as one treats one-body observables such as heat, flux, and dose in the transport calculations with the nuclear data. However, as stressed in this paper, the quantities beyond onebody observable are often required in many new fields. The treatment of the event generator mode in PHITS proposed in this paper might be a direction to fulfil the new fields requirements.

\section{References}

1. H. Iwase, K. Niita, T. Nakamura, J. Nucl. Sci. Technol. 39, 1142 (2002).

2. Y. Nara, N. Otuka, A. Ohnishi, K. Niita, S. Chiba, Phys. Rev. C 61, 024901 (2000).

3. K. Niita, S. Chiba, T. Maruyama, H. Takada, T. Fukahori, Y. Nakahara, A. Iwamoto, Phys. Rev. C 52, 2620 (1995).

4. J.F. Briesmeister et al., MCNP: General Monte Carlo N-Particle Transport Code, LANL Report, LA-12625-M (1997).

5. Y. Iwamoto, K. Niita, Y. Sakamoto, T. Sato, N. Matsuda (these proceedings). 